\title{
A proposed sustainability index for synthesis plans based on input provenance and output fate: application to academic and industrial synthesis plans for vanillin as a case study
}

\author{
John Andraos
}

\author{
Full Research Paper \\ Address: \\ CareerChem, 504-1129 Don Mills Road, Toronto, ON, M3B 2W4, \\ Canada \\ Email: \\ John Andraos - c1000@careerchem.com \\ Keywords: \\ Borda count; green chemistry; input enthalpic energy; process mass \\ intensity; poset dominance analysis; Rowan solvent greenness index; \\ sacrificial reagent; sustainability; sustainable chemistry \\ Beilstein J. Org. Chem. 2020, 16, 2346-2362. \\ https://doi.org/10.3762/bjoc.16.196 \\ Received: 04 October 2019 \\ Accepted: 02 September 2020 \\ Published: 25 September 2020 \\ This article is part of the thematic issue "Green chemistry II". \\ Associate Editor: L. Vaccaro \\ (C) 2020 Andraos; licensee Beilstein-Institut. \\ License and terms: see end of document.
}

\begin{abstract}
This paper describes a sustainability index (SI) as a quantitative measure of "sustainability" applicable to synthesis plans based on the provenance of input materials and energy sources and the fate of output waste products. The index is computed as the rootmean-square average of the following four parameters: mass fraction of valorized inputs $\left(F_{V I}\right)$, mass fraction of valorized outputs $\left(F_{V O}\right)$, mass fraction of valorized target product $\left(F_{V P}\right)$, and input enthalpic energy fraction arising from renewable energy sources $\left(F_{R E}\right)$. Valorized input materials originate from renewable, recycled, or reclaimed sources. Valorized output materials are destined for recycling or reclaiming so that they may be used in the same or other chemical processes. Valorized target product refers to that portion of the target product that is actually used for its intended purpose. Renewable energy sources are defined as originating from hydroelectric, wind, solar, geothermal, and biomass sources. The computation of SI is illustrated for 22 synthesis plans of the high commodity flavour ingredient vanillin from biofermentation, chemical synthesis, and solvent extraction processes. In addition, these plans are compared and ranked according to Borda count and poset (partially ordered set) pairwise dominance analyses using the following attributes: process mass intensity (PMI), sacrificial reagent (SR) consumption, input enthalpic energy (IEE) consumption, Rowan solvent greenness index (RSGI), and sustainability index (SI).
\end{abstract}

\section{Introduction}

The words "sustainable" and "sustainability" are nowadays routinely used throughout common speech and the popular press, including published modern chemistry literature, when discussing topics related to pressing issues such as preservation of the environment, climate change, and resource management. However, in all of this enormous volume of information avail- 
able in the popular press, corporate mission statements, and scientific literature there does not exist an agreed consensusbased and widely used quantitative definition of what these words actually mean. A recent article published in Chemical and Engineering News in 2019 [1] nicely highlighted the problem in the context of distinguishing the terms green chemistry from sustainable chemistry. It was noted that "the term sustainable chemistry has been introduced more recently and possesses countless definitions put forth by individuals, companies, trade associations, non-profit organizations, and governmental entities". Also noted was the "key need [to come up with] a standardized approach for assessing the sustainability of chemical processes or products", and the need for "better information on product content throughout the supply chain and more complete data on the health and environmental impacts of chemicals throughout their life-cycle" in order for "stakeholders [to] make informed decisions that compare the sustainability of various products". A literature search on the subject of sustainability metrics in chemistry journals revealed a few publications that addressed the problem of quantifying and measuring what sustainability is [2-10]. Most of the discussions revolved around extended thermodynamics analysis, energy consumption, and energy resource considerations from fossil-fuel derived and renewable sources. Dutch and Belgian chemical engineers proposed an extended thermodynamic analysis considering exergy and lost work to address the sustainability potential of the chemical process industry [2-4]. Horvath and co-workers put forth the following sustainability metrics defined for biomass-based carbon chemicals using ethanol equivalent as a common basis: sustainability value of resource replacement, sustainability value of the fate of waste, and sustainability indicator [5-7]. Sikdar defined sustainability as the interplay of three domains: economic aspects, environmental aspects, and social aspects [8]. Sheldon and Sanders defined sustainability metrics for the production of chemicals from renewable biomass in terms of four criteria: material and energy efficiency, land use, and process economics [9]. Very recently, Egyptian and Lebanese scientists put forward an industrial environmental index obtained from process, environmental health and safety, and life cycle assessment metrics to assess the sustainability of industrial solvent-based processes [10].

Continuing our goal over the last decade of developing practical and easy-to-understand metrics that can be used by any chemist or chemical engineer, in this work we introduce a quantitative description of sustainability that is directly applicable to assess synthesis plans. Synthesis plans represent the heart and soul of what chemists create in the laboratory and chemical engineers scale up and optimize in the chemical plant. Hence, any chance of developing a quantitative definition of sustain- ability that addresses the needs and concerns already pointed out must focus on assessing the performance of synthesis plans according to some set of measurable parameters. This is a very important aspect in the evolution of scientific ideas that begin with qualitative statements often worded in fuzzy general language, but then develops into concrete statements written in the language of mathematics that make explicit what parameters need to be measured, how they are related to one another, and what outcome scenarios are predicted from them. The net result is that vagaries are removed from the discussion and hence the subject is presented in a rigorous and understandable format that is ultimately taken seriously. Since it is evident that synthesis plans begin with input resources and ultimately produce output products, it then becomes necessary to analyze both the origins of all inputs and the fate of all outputs in order to determine the sustainability potential of a given synthesis plan. In order to achieve our objective, we decided to begin from two already established main ideas. The first is the comparison of the rate of depletion of each resource (material or energy) used in a synthesis plan versus the rate of that resource's renewal as pointed out by Horvath and co-workers [5-7]. Clearly, sustainability is possible if the rate of renewal exceeds the rate of depletion. The second is the concept of "provenance" borrowed from the authentication of objects of art as genuine by art dealers and museum curators during the selling and purchasing of them at auctions. The provenance of an object of art potentially links it to its true creator. Obtaining provenance constitutes amassing traceable hard evidence that links the original artist and his or her work via the chain of its ownership through time from its creation to the present day. Provenances are necessary to establish the authenticity of a piece of art and thus distinguish it from a forgery. The concept of provenance or tracing has also been applied in other fields including computer science, data integrity management, petrology, archaeology, seed preservation, food authenticity, and palaeontology [11]. In the context of synthesis plans, it is possible to apply the concept of provenance to the origins of all material and energy resources used in a given plan in order to distinguish whether that resource originated from a renewable or non-renewable source. By the same reasoning, it is also possible to trace the fate of all waste outputs of a given plan in order to distinguish whether they will end up as useable or non-usable waste. From this discussion it becomes obvious that the success of tracing both provenance of inputs and fate of outputs involved in a synthesis plan in order to estimate the degree of its sustainability is based entirely on the full disclosure of supply chains and end-of-life chains throughout the chemistry enterprise. Such open access and transparent information, however, necessarily exposes vulnerabilities in those chains such as privacy with respect to business-to-business dealings and general proprietary protection that may not be comfortable to accept or possible to reveal 
to producers, suppliers, vendors, and end users. These concerns are at the root cause of much of the vagueness and trepidation associated with the practical application of sustainability as already pointed out earlier [1]. Nevertheless, in this work we provide a framework that can be implemented to estimate the degree of sustainability of synthesis plans following these ideas once such information is made available whether internally for a privileged few or externally for all to see. With this view in mind, in the next sections we show a step-by-step development of our methodology and then apply it to the assessment of 22 synthesis plans of the high commodity flavour ingredient vanillin.

\section{Methodology}

We begin the development of a quantitative description of sustainability applied to synthesis plans by starting with the mathematical statement of the law of mass balance given by Equation 1.

$$
M_{\text {total inputs }}=M_{\text {total outputs }}
$$

where the $M$ quantities refer to masses in grams. The left-hand side of Equation 1 will be governed by the provenance of input materials and the right-hand side will be governed by the fate of output materials. Hence, the mass of total inputs can be subdivided into two parts: mass of valorized inputs $\left(M_{V I}\right)$ and mass of non-valorized inputs $\left(M_{N V I}\right)$ according to Equation 2.

$$
M_{\text {total inputs }}=M_{V I}+M_{N V I}
$$

In this formulation, valorized inputs are defined as those that are derived from sources such that their rate of renewal is greater than or equal to their rate of depletion, and nonvalorized inputs are defined as those that are derived from sources where the converse rate condition is true. Specifically, inputs derived from renewable or recycled sources such as biomass, scrap metals, or retrieved byproducts from other processes are considered valorized, and inputs derived from nonrenewable sources such as fossil fuels and virgin mineral ores are considered non-valorized. The definition of $F_{V I}$ used here extends that of renewables intensity [12]. Similarly, the mass of total outputs may be subdivided into three parts: waste mass of valorized outputs $\left(W_{V O}\right)$, waste mass of non-valorized outputs $\left(W_{N V O}\right)$, and mass of target product $\left(M_{\text {product }}\right)$ as shown in Equation 3.

$M_{\text {total outputs }}=W_{\text {total }}+W_{\text {product }}=W_{V O}+W_{N V O}+M_{\text {product }}$

Valorized waste outputs are those that may be recycled or reclaimed for use in the same synthesis plan or other unrelated synthesis plans if they are sold to other chemical enterprises in the chemical commodity supply chain. These may include reaction and work-up solvents or reaction byproducts than can be chemically converted to starting materials, or byproducts that can be reclaimed for use in other chemical processes. Nonvalorized waste outputs are those that will end up as "dead waste" whether or not they undergo treatment before release into the four main environmental compartments of air, water, soil, and sediment. Based on the definition of variables in Equations 1,2 , and 3 we can then define the following key parameters. The process mass intensity (PMI) [13] is defined according to Equation 4.

$$
P M I=\frac{M_{V I}+M_{N V I}}{M_{\text {product }}}=\frac{M_{\text {total inputs }}}{M_{\text {product }}}
$$

The mass fractions of valorized inputs $\left(F_{V I}\right)$ and valorized waste outputs $\left(F_{V O}\right)$ are defined according to Equation 5 and Equation 6.

$$
\begin{gathered}
F_{V I}=\frac{M_{V I}}{M_{\text {total inputs }}}=\frac{M_{V I}}{M_{V I}+M_{N V I}} \\
F_{V O}=\frac{W_{V O}}{W_{V O}+W_{N V O}}
\end{gathered}
$$

We can also define a mass fraction of valorized target product $\left(F_{V P}\right)$ according to Equation 7 that describes the proportion of target product of a synthesis plan that is actually used for its intended purpose. This takes into account the end-of-life stage of the life cycle of the target product where part of it will end up as "dead waste".

$$
F_{V P}=\frac{M_{\text {product }}-M_{\text {product }}^{*}}{M_{\text {product }}}
$$

where $M_{\text {product }}^{*}$ is the mass of target product that is destined to be wasted. For example, if the target product of a synthesis plan is a pharmaceutical compound a certain fraction of its manufacture will be used as intended by patients; however, there will be a remaining fraction that will be destined as non-usable waste via natural excretion by the human body and more importantly via disposal by pharmacies when the medicine passes its recommended safe expiry date. The estimation of $F_{V P}$ will always rely on significant assumptions and guesswork since there are no proper centralized data kept for tracking end-of-life waste of any product manufactured in the chemical industry. Hence, for the purposes of calculating SI, estimating $M_{\text {product }}^{*}$ is the weakest link. 
Having described the three mass fractions related to the provenance of input materials and fate of output materials, we may now examine the energy source provenance for conducting all heating and cooling operations involved in all reaction steps in a synthesis plan. We define a total input enthalpy energy, $(I E E)_{\text {total }}$, as shown in Equation 8 where it is divided into renewable and non-renewable energy sources.

$$
(I E E)_{\text {total }}=(I E E)_{\text {renewable }}+(I E E)_{\text {non-renewable }}
$$

The explicit formulation of (IEE) total is shown in Equation 9 where it is obtained as a sum of all energy consumptions as a result of heating and cooling over all input materials used in a synthesis plan above or below a reference state representing the ambient temperature and pressure conditions of $298 \mathrm{~K}$ and $1 \mathrm{~atm}$, respectively. Temperature deviations are governed by temperature dependent heat capacity relationships for each substance, and pressure deviations are governed by volumetemperature relationships according to some specified equation of state. In practice, the contribution to IEE from temperature deviations far exceeds that from pressure deviations for reaction pressures below $100 \mathrm{~atm}$. In cases where the reaction pressure exceeds $100 \mathrm{~atm}$, the Redlich-Kwong equation of state formalism [14] was used in this work for computing IEE values.

$$
\begin{aligned}
& (I E E)_{\text {total }}=\sum_{j=1}^{\text {total inputs }}(I E E)_{j}= \\
& \sum_{j=1}^{\text {total inputs }}\left[\text { moles }_{j}\left(\begin{array}{l}
\int_{298}^{T_{r x n}} C_{p, j}(T) d t+\int_{0}^{0}\left[V-T\left(\frac{\partial V}{\partial T}\right)_{p}\right]_{T=298} d p \\
+\int_{0}^{p_{r x n}}\left[V-T\left(\frac{\partial V}{\partial T}\right)_{p}\right]_{T=T_{r x n}} d p
\end{array}\right)\right]
\end{aligned}
$$

Similar to the mass fractions defined in Equations 5-7, we can define an analogous input enthalpic energy fraction arising from renewable energy sources $\left(F_{R E}\right)$ as shown in Equation 10. This definition is similar to renewability index proposed earlier [15]

$$
F_{R E}=\frac{(I E E)_{\text {renewable }}}{(I E E)_{\text {total }}}
$$

In the present formalism we define the following energy sources as renewable: hydroelectric, solar, wind, geothermal, and biofuels; and the following energy sources as non-renewable: coal, other fossil-fuels such as petroleum and natural gas, and nuclear. Furthermore, following recently published energy mix data $[16,17]$ we set $F_{R E}=0.35$ for all synthesis plans that were published on or after the year 2000 and $F_{R E}=0$ for all synthesis plans that were published before 2000 . We chose the year 2000 as an arbitrary boundary time frame since it marked the beginning of the 21 st century when ideas of sustainability began to take root in the general societal consciousness.

Taking into account the four fractional values $F_{V I}, F_{V O}, F_{V P}$, and $F_{R E}$ we can define an overall sustainability index (SI) which is the root-mean-square average of these four fractional quantities as shown in Equation 11.

$$
\begin{aligned}
S I & =\frac{\sqrt{\left(F_{V I}\right)^{2}+\left(F_{V O}\right)^{2}+\left(F_{V P}\right)^{2}+\left(F_{R E}\right)^{2}}}{\sqrt{4}} \\
& =\frac{1}{2} \sqrt{\left(F_{V I}\right)^{2}+\left(F_{V O}\right)^{2}+\left(F_{V P}\right)^{2}+\left(F_{R E}\right)^{2}}
\end{aligned}
$$

Since each of these fractions has values ranging between 0 and 1 , then the magnitude of SI will also have a value ranging between 0 and 1 . This mathematical formalism conveniently allows the writing down of a quantitative definition of sustainability applicable to synthesis plans. A given synthesis plan can therefore be said to be completely "sustainable" if the following conditions are satisfied: $F_{V I}=1, F_{V O}=1, F_{V P}=1, F_{R E}=1$, and $\mathrm{SI}=1$. Conversely, a given synthesis plan can be said to be completely "unsustainable", if the following conditions are satisfied: $F_{V I}=0, F_{V O}=0, F_{V P}=0, F_{R E}=0$, and SI $=0$.

Having this new sustainability metric in hand, we can then use it along with process mass intensity (PMI), sacrificial reagent (SR) consumption, input enthalpic energy (IEE), and Rowan solvent greenness index (RSGI) as key attributes to rank any kind of synthesis plan or chemical process. PMI and IEE have already been defined in Equations 4 and 9. SR consumption defined in Equation 12 quantifies the mass fraction of reagents whose atoms do not contribute to the chemical structure of the final target product of the synthesis plan. Following the concept of atom economy [18], this attribute is important in designing syntheses that maximize the use of all atoms in input reagents towards the final product structure while minimizing waste byproducts as a consequence of producing the intended intermediates over the course of the synthesis sequence. The RSGI [19] given in Equation 13 is a convenient metric that is used to quantify the relative environmental, toxicological, and safetyhazard impacts of solvents used in reaction, work-up, and purification procedures. It is defined using an overall solvent index (OSI) that scales between 0 and 12 spanning the benign solvent water to the non-benign solvent benzene. Equation 14 and Equation 15 show the explicit dependence of OSI on 15 physical, toxicological, and hazard parameters.

$$
S R=\frac{\sum \text { mass sacrificial reagents }}{\sum \text { total mass reagents }}
$$




$$
R S G I=\sum_{i} m_{i}\left(O S I_{12}\right)_{i}
$$

where $m_{i}$ is the mass of solvent $i$ and $\mathrm{OSI}_{12}$ is defined as a normalized quantity over a set of solvents as shown in Equation 14.

$$
\left(O S I_{12}\right)_{i}=12\left(\frac{O S I_{i}-O S I_{\min }}{O S I_{\max }-O S I_{\min }}\right)
$$

where OSI $_{\min }$ and OSI $_{\max }$ are the minimum and maximum values of OSI for a set of solvents and $\operatorname{OSI}_{i}$ for a given solvent $i$ is given by Equation 15 .

$$
\begin{aligned}
O S I_{i}= & 2\left(M_{O E L, i}+M_{L D 50, i}+M_{L C 50, i}\right)+M_{G W P, i} \\
& +M_{S E P, i}+M_{O D P, i}+M_{A B P, i}+M_{B C P, i}+M_{P E R, i} \\
& +M_{\text {soil }, i}+M_{\text {half life }, i}+M_{\text {aqua }, i}+M_{Q-\text { phrase }, i} \\
& +M_{S D, i}+M_{F P, i}
\end{aligned}
$$

where the metric parameters $(M)$ cover occupational exposure limit (OEL, ppm), $\mathrm{LD}_{50}$ (ingestion toxicity, $\mathrm{mg} / \mathrm{kg}$ ), $\mathrm{LC}_{50}$ (inhalation toxicity, $\mathrm{g} \mathrm{m}^{-3}$ for $4 \mathrm{~h}$ ), global warming potential (GWP, unitless), smog formation potential (SFP, unitless), ozone depletion potential (ODP, unitless), acidity-basicity potential (ABP, unitless), bioconcentration potential (BCP, unitless), persistence potential (PER, unitless), soil sorption coefficient (soil, $K_{\mathrm{oc}}$ ), half-life of solvent in environment (half-life, h), aquatic toxicity to fish (aqua, $\mathrm{mg} / \mathrm{L}$ for $96 \mathrm{~h}$ ), Q-phrase potential (Q-phrase, unitless), skin dose (SD, mg), and flash point (FP, degrees $\mathrm{K}$ ). Table 1 shows a revised and expanded formatted listing of normalized OSI values for various solvents used in the chemical industry.

In our past work [20] describing a presentation of a "standardized process green synthesis report" for chemical syntheses of pharmaceutical compounds we were able to demonstrate the Borda count [21-24] and poset (partially ordered set) pairwise dominance [25] ranking algorithms based on the four attributes PMI, SR, IEE, and RSGI. In the present work, we can now add SI as a fifth key attribute as part of those ranking algorithm analyses which takes into account sustainability potential as well as material and energy consumption and environmental and safety-hazard impacts. In order to demonstrate these ideas, we chose to examine 22 academic and industrial synthesis plans for the manufacture of $1 \mathrm{~kg}$ of vanillin since this high

\begin{tabular}{|c|c|}
\hline $\mathrm{OSI}_{12}{ }^{\mathrm{a}}$ & solvent \\
\hline 12.000 & benzene \\
\hline 10.597 & chlorobenzene \\
\hline 10.350 & aniline \\
\hline 10.150 & toluene \\
\hline 10.130 & nitrobenzene \\
\hline 10.077 & pyridine \\
\hline 9.885 & triethylamine \\
\hline 9.774 & o-xylene \\
\hline 9.773 & $p$-xylene \\
\hline 9.703 & $m$-xylene \\
\hline 9.677 & 1,2-dichlorobenzene \\
\hline 9.653 & 1,2-dichloroethane \\
\hline 9.324 & formaldehyde \\
\hline 9.048 & $n$-hexane \\
\hline 8.879 & methylcyclohexane \\
\hline 8.777 & cyclohexane \\
\hline 8.534 & 2-methyltetrahydrofuran \\
\hline 8.451 & carbon tetrachloride \\
\hline 8.421 & diethyl ether \\
\hline 8.365 & dimethylacetamide \\
\hline 8.309 & acetic anhydride \\
\hline 8.090 & chloroform \\
\hline 8.016 & carbon disulfide \\
\hline 7.985 & tetrahydrofuran \\
\hline 7.927 & acetic acid \\
\hline 7.875 & tert-butanol \\
\hline 7.852 & cyclopentyl methyl ether \\
\hline 7.734 & petroleum ether \\
\hline 7.727 & 1,4-dioxane \\
\hline 7.647 & isopropyl acetate \\
\hline 7.603 & acetonitrile \\
\hline 7.597 & ethyl acetate \\
\hline 7.592 & $p-N, N$-dimethyltoluidine \\
\hline 7.429 & $n$-heptane \\
\hline 7.425 & trifluorotoluene \\
\hline 7.402 & dimethylformamide \\
\hline 7.365 & methyl tert-butyl ether \\
\hline 7.323 & hexamethylphosphoric triamide \\
\hline 7.278 & methyl ethyl ketone \\
\hline 7.129 & dichloromethane \\
\hline 7.074 & acetone \\
\hline 6.966 & 1-heptanol \\
\hline 6.952 & 1-propanol \\
\hline 6.732 & methyl propionate \\
\hline 6.719 & isopropanol \\
\hline 6.706 & trichloroethylene \\
\hline 6.644 & $n$-butanol \\
\hline 6.547 & nitromethane \\
\hline 6.505 & $n$-pentane \\
\hline
\end{tabular}
commodity flavour chemical is ideally suited to the present investigation owing to its varied methods of synthesis spanning classical chemical synthesis, biofermentation, and solvent ex-
Table 1: Revised summary of overall solvent index (OSI) for various organic solvents used in the pharmaceutical industry. 
Table 1: Revised summary of overall solvent index (OSI) for various organic solvents used in the pharmaceutical industry. (continued)

\begin{tabular}{|c|c|}
\hline 6.108 & methyl formate \\
\hline 5.905 & methyl acetate \\
\hline 5.859 & ethylene glycol monomethyl ether \\
\hline 5.772 & isoamyl alcohol \\
\hline 5.672 & amyl acetate \\
\hline 5.620 & isoamyl acetate \\
\hline 5.593 & sec-butanol \\
\hline 5.495 & $N$-methylpyrrolidinone \\
\hline 5.426 & methanol \\
\hline 5.360 & isobutyl acetate \\
\hline 5.352 & anisole \\
\hline 5.298 & tert-amylalcohol \\
\hline 5.268 & cyclopentanone \\
\hline 5.210 & trifluoroacetic acid \\
\hline 5.110 & isopropyl ether \\
\hline 5.106 & 1-octanol \\
\hline 4.773 & ethanol \\
\hline$\overline{4.751}$ & ethylene glycol \\
\hline$\overline{4.538}$ & thionyl chloride ${ }^{b}$ \\
\hline 4.535 & dimethoxymethane \\
\hline 4.474 & isooctane \\
\hline 4.224 & dimethyl carbonate \\
\hline$\overline{4.182}$ & glycol diacetate \\
\hline$\overline{3.908}$ & diglyme \\
\hline 3.795 & sulfolane \\
\hline 3.679 & $\mathrm{sCO}_{2}$ \\
\hline 3.266 & ethylene glycol dimethyl ether \\
\hline 3.250 & triethylene glycol monomethyl ether \\
\hline$\overline{3.027}$ & propylene carbonate \\
\hline 2.803 & dimethyl sulfoxide \\
\hline$\underline{2.485}$ & propylene glycol \\
\hline 2.233 & dimethylisosorbide \\
\hline 0.000 & water \\
\hline
\end{tabular}

aUnderlined entries indicate benign performance $\left(O S I_{12} \leq 5\right)$; italicized entries indicate intermediate performance $\left(5<\mathrm{OSI}_{12}<8\right)$; and boldformatted entries indicate worst performance $\left(O S I_{12} \geq 8\right)$. bMissing $\mathrm{LC}_{50}$ (oral), $\mathrm{LD}_{50}$ (inhalation), and aquatic toxicity data; used as a dual reagent and solvent in industrial syntheses of acid chlorides from carboxylic acids.

traction procedures from the natural source vanilla beans. We chose these particular examples from the literature since they had the most detailed experimental procedures from which the set of discussed metrics could be reliably determined and then ranked. Figure S1 found in Part 1 of Supporting Information File 1 shows all of the schemes pertaining to the 22 synthesis plans listed in alphabetical order along with temperature and pressure conditions for each reaction step. Table 2 summarizes the same alphabetized list showing the plan codes, starting materials used, and type of chemical process employed. Four plans involved biofermentation from D-glucose, ferulic acid, or isoeugenol [26-30]; five plans involved chemical synthesis from wood-derived starting materials (lignosulfonic acid liquor or sawdust) [31-37]; seven plans involved chemical synthesis from either fossil-fuel or natural product-derived starting materials (guaiacol, eugenol, isoeugenol, and 4-hydroxybenzaldehyde) [38-43]; and five plans involved solvent extraction procedures either by percolation, Soxhlet, or supercritical fluid methods using cured vanilla beans that were either whole or were cut up as starting material [44-46].

\section{Results and Discussion Sustainability of vanillin plans}

The exercise of partitioning input materials according to their provenance and output materials according to their fate is extremely challenging because it requires a completely transparent knowledge and access to the entire network supply and end-of-life chains that constitute the chemical industry enterprise. In practice, when reading experimental sections in journal publications and patents, one has limited or no access to such background knowledge even though authors may disclose the names of chemical suppliers of the starting materials they used in their own work. It is a fair comment to say that authors themselves may not know or care to know the chain of supply of those starting materials so long as they have them in hand in a sufficiently pure condition to carry out their own research agenda. In any event, the task of estimating a quantitative measure of sustainability of any given synthesis plan according to the formalism of Equation 11 will require significant assumptions to be made. For the purpose of our work, which is meant to be illustrative only in the numerical methods employed, we implemented the following assumptions in the calculation of SI for all 22 plans of vanillin:

(1) If ethanol is used as an input material then $10 \%$ of it was assumed to originate from renewable sources (i.e., biomass), if the publication is dated after 1990 since that is the approximate time frame when biofuels were made widely available in the market.

(2) Water was considered a renewable input material due to the circulating global hydrological cycle.

(3) Mineral salts, metal-derived catalysts, and all non-aqueous and non-biologically derived materials from fossil fuels or ores were considered non-renewable inputs since their rate of renewal occur on geological time scales that are several orders of magnitude longer than organism time scales.

(4) Lignosulfonic acid liquor and sawdust were considered renewable inputs since they ultimately originate from trees. Ferulic acid and D-glucose were considered renewable inputs 
Table 2: Summary of plan codes, starting materials, and process types for 22 synthesis plans of vanillin.

\begin{tabular}{|c|c|c|c|}
\hline Alphabetized list of vanillin synthesis plans & plan code & starting material & process \\
\hline Borregaard synthesis & p1 & lignosulfonic acid liquor & chemical synthesis \\
\hline Collins chemical & p2 & isoeugenol & chemical synthesis \\
\hline Eilks Pt 1 & p3 & isoeugenol & chemical synthesis \\
\hline Eilks Pt 2 & $\mathrm{p} 4$ & isoeugenol & chemical synthesis \\
\hline Faith & p5 & lignosulfonic acid liquor & chemical synthesis \\
\hline Frost & p6 & D-glucose & biofermentation \\
\hline Givaudan-Roure & p7 & ferulic acid & biofermentation \\
\hline Haarmann and Reimer & p8 & isoeugenol & biofermentation \\
\hline Hibbert & p9 & lignosulfonic acid liquor & chemical synthesis \\
\hline $\mathrm{Ji}$ & p10 & guaiacol & chemical synthesis \\
\hline Lampman & p11 & sawdust & chemical synthesis \\
\hline Lesage-Meesen & p12 & ferulic acid & biofermentation \\
\hline Mayer & p13 & eugenol & chemical synthesis \\
\hline Mexican group SFE & p14 & cured vanilla pods & solvent extraction \\
\hline Mottern & p15 & guaiacol & chemical synthesis \\
\hline Ontario Paper Co. & p16 & lignosulfonic acid liquor & chemical synthesis \\
\hline percolation extraction cut & p17 & cured vanilla pods & solvent extraction \\
\hline percolation extraction whole & p18 & cured vanilla pods & solvent extraction \\
\hline Sorensen-Mehlum & p19 & sawdust & chemical synthesis \\
\hline Soxhlet extraction cut & p20 & cured vanilla pods & solvent extraction \\
\hline Soxhlet extraction ground & $\mathrm{p} 21$ & cured vanilla pods & solvent extraction \\
\hline Taber & p22 & 4-hydroxybenzaldehyde & chemical synthesis \\
\hline
\end{tabular}

since they originate from sugar beet biomass [47] and other cellulosic biomass feedstocks, respectively. Isoeugenol and eugenol were chosen to originate from the natural product clover oil since $80 \%$ by weight of this essential oil is eugenol [48], and isoeugenol is directly obtained from eugenol by basecatalyzed isomerization.

(5) Oxygen from air was considered a renewable input for the oxidation of lignosulfonic acid liquors since it is produced as a natural waste byproduct of photosynthesis.

(6) Spent vanilla beans were considered reusable waste since they can be composted into biomass.

(7) Supercritical carbon dioxide was considered reusable waste since it can be recycled in the same solvent extraction process via continuous pressurization and depressurization cycles.

(8) A renewable energy source contribution of $35 \%\left(F_{R E}=\right.$ 0.35 ) was assigned, if the publication is dated on or after the year 2000 for reasons discussed earlier.

(9) Since vanillin is mainly used as a food flavouring agent, $70 \%$ of it was assumed to be actually used in the food industry
$\left(F_{V P}=0.7\right)$ and $30 \%$ of it will end up as part of the food waste stream which mirrors recent statistics [49-53] that assert that about a third of all foodstuffs produced will end up wasted along supply chains and by end-of-use consumers.

In order to facilitate computation of SI an Excel-based calculator was developed that can be used for any synthesis plan once all inputs and outputs are identified (see Supporting Information File 2). Firstly, for a given synthesis plan, all scaled masses of input and output materials are entered for the production of $1 \mathrm{~kg}$ of vanillin. Scaled mass data for each plan are found in Part 2 of Supporting Information File 1. Secondly, valorized input and output materials are selected according to the assumptions listed above. Finally, $F_{R E}$ is set to 0.35 or 0 for plans published after or before the year 2000, respectively and $F_{V P}$ is set at 0.7 . Figure 1 shows radial diamond diagrams depicting the four fractions for the leading eight most sustainable plans representing biofermentation and solvent extraction methods. The main reason for their high SI scores is the combined high values of $F_{V I}$ and $F_{V O}$ close to unity in each case. For the solvent extraction procedures low values of $F_{V I}$ are found if the mass ratio of solvent to vanilla bean is very high as is the case for the percolation extraction cut, Soxhlet extraction cut, and Mexican group SFE plans. For the case of 

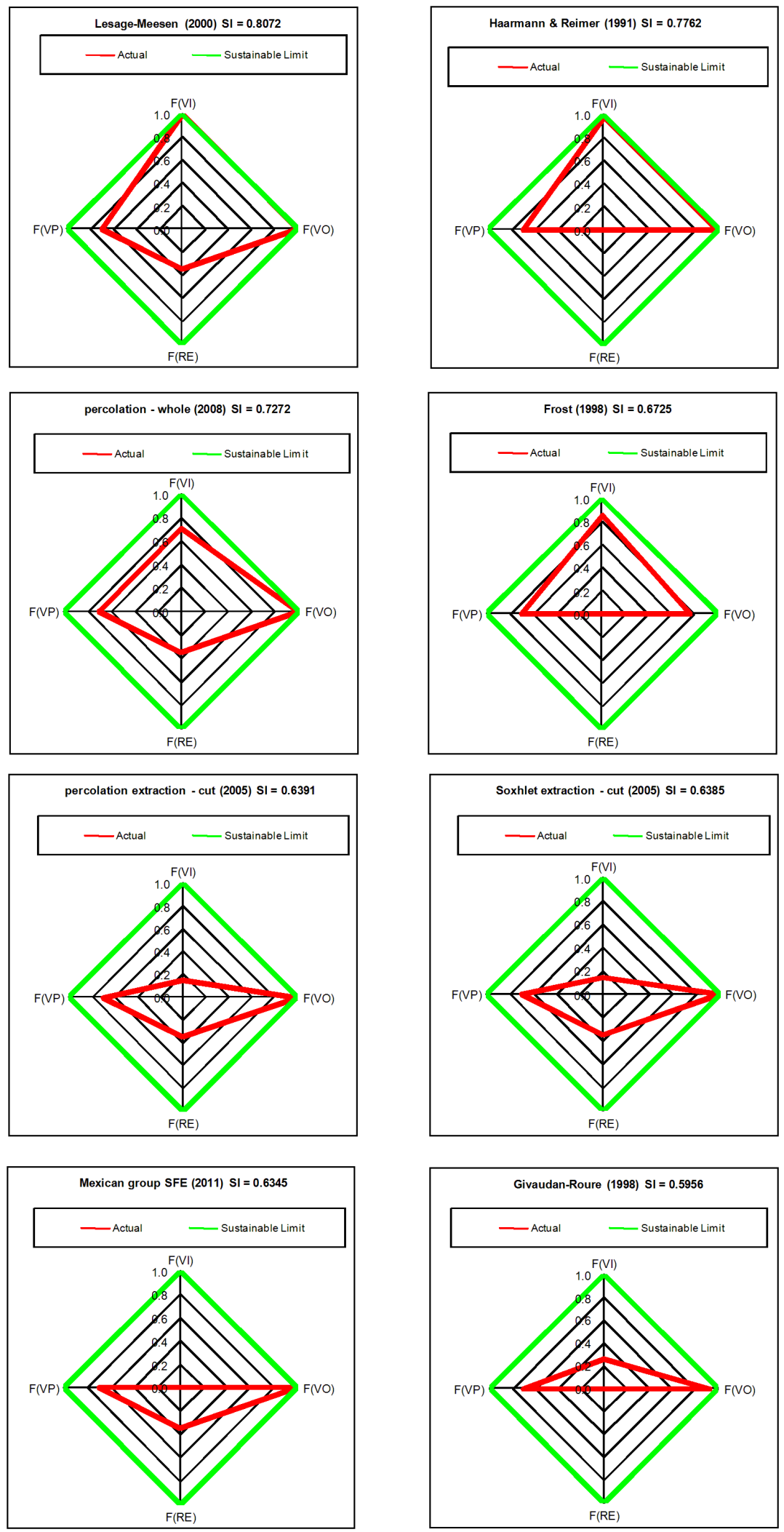

Figure 1: Radial diamond diagrams illustrating the sustainability index (SI) computed based on $F_{V I}, F_{V O}, F_{V P}$, and $F_{R E}$ for the top eight scoring vanillin synthesis plans. 
supercritical carbon dioxide used as an extraction solvent, the main drawback is that industrial carbon dioxide is classified as originating from a non-renewable mineral resource (mainly calcium carbonate) and that the mass ratio of $\mathrm{sCO}_{2}$ solvent to vanilla beans is $435: 1$. Synthesis plans based on chemical syntheses from lignosulfonic acid liquor, guaiacol, eugenol and isoeugenol have $F_{V O}$ values of 0 since none of their solvent and waste byproducts are reuseable. However, $F_{V I}$ values for these syntheses range between 0 (Eilks - Pt 2) and 0.898 (Borregaard) with an average value of 0.414 over 14 plans. With respect to renewable energy sources, only nine out of the 22 plans were assigned a value of 0.35 for $F_{R E}$ since their publications appeared on or after the year 2000.

Table 3 summarizes numerical results of the 5 attributes PMI, SR, IEE, RSGI, and SI for all 22 synthesis plans of vanillin. From this list we observe that chemical syntheses tend to have lower PMI values and biofermentation and solvent extraction methods tend to have higher PMI values due to higher solvent consumption in the latter group. Ten out of the 22 plans had SR values of 0 and the rest of the plans had higher values in the range 1.2 to 92, except for the Eilks - Pt 1 and Lampman plans that used isoeugenol and sawdust, respectively, as starting materials. With respect to energy consumption, the two percolation extraction procedures using cut or whole cured vanilla beans with no heating of solvents had the lowest IEE value of 0 . The other plans had significant energy demands for various reasons. The methods starting from lignosulfonic acid liquors were conducted under elevated temperature and pressure conditions of $200{ }^{\circ} \mathrm{C}$ and $100 \mathrm{~atm}$. The SFE method using a high amount of $\mathrm{sCO}_{2}$ required pressurization conditions of about $200 \mathrm{~atm}$. The biofermentation methods, though they were conducted under biologically ambient conditions of $37{ }^{\circ} \mathrm{C}$ and $1 \mathrm{~atm}$, had high IEE values as a result of the high mass of aqueous nutrient broths required for their operation relative to the small mass of starting materials. For example, the most sustainable Lesage-Meesen plan with the highest SI value of 0.8072 had an IEE value of $939,475 \mathrm{~kJ} / \mathrm{kg}$ owing to the $1254: 1$ combined aqueous nutrient medium-to-substrate ratio. With respect to solvent impacts, the Givaudan-Roure, Lesage-Meesen, and Haarmann and Reimer biofermentation procedures had RSGI values of 0 . The only other plan with a zero solvent impact was the improved lignosulfonate method of Borregaard carried out in aqueous solution. The traditional chemical synthesis methods had more of a solvent impact due to the use of the following solvents listed in descending order of impact according to OSI values given in Table 1: benzene (12.000), aniline (10.350), toluene (10.150), nitrobenzene (10.130), cyclohexane (8.777), diethyl ether

Table 3: Summary of computed values for five attributes for 22 synthesis plans of vanillin.

\begin{tabular}{|c|c|c|c|c|c|}
\hline $\begin{array}{l}\text { Alphabetized list of vanillin } \\
\text { synthesis plans }\end{array}$ & PMI (kg/kg) & $\mathrm{SR}(\mathrm{kg} / \mathrm{kg})$ & IEE (kJ/kg) & RSGI (kg/kg) & $\mathrm{SI}$ \\
\hline Borregaard synthesis & 84 & 8.6 & 169046 & 0 & 0.5756 \\
\hline Collins chemical & 38 & 1.2 & 50440 & 38 & 0.4603 \\
\hline Eilks Pt 1 & 1712 & 341 & 17309 & 4492 & 0.4866 \\
\hline Eilks Pt 2 & 147 & 0 & 2881 & 673 & 0.3907 \\
\hline Faith & 50 & 0 & 29286 & 6 & 0.4519 \\
\hline Frost & 15973 & 92 & 578763 & 16369 & 0.6725 \\
\hline Givaudan-Roure & 21 & 0 & 795 & 0 & 0.5956 \\
\hline Haarmann and Reimer & 1159 & 0 & 9388 & 0 & 0.7762 \\
\hline Hibbert & 803 & 71 & 1005329 & 3768 & 0.4207 \\
\hline $\mathrm{Ji}$ & 3843 & 45 & 325200 & 24670 & 0.3914 \\
\hline Lampman & 32540 & 9845 & 2398980 & 112106 & 0.3676 \\
\hline Lesage-Meesen & 19434 & 30 & 939475 & 0 & 0.8072 \\
\hline Mayer & 26 & 10 & 20989 & 163 & 0.3704 \\
\hline Mexican group SFE & 7513 & 0 & 1678005 & 27578 & 0.6345 \\
\hline Mottern & 125 & 0 & 2181 & 277 & 0.4551 \\
\hline Ontario Paper Co. & 730 & 32.5 & 688521 & 2101 & 0.4689 \\
\hline percolation extraction cut & 831 & 0 & 0 & 3727 & 0.6391 \\
\hline percolation extraction whole & 1028 & 0 & 0 & 1579 & 0.7272 \\
\hline Sorensen-Mehlum & 563 & 29.5 & 653838 & 1952 & 0.4171 \\
\hline Soxhlet extraction cut & 582 & 0 & 50219 & 2644 & 0.6385 \\
\hline Soxhlet extraction ground & 5332 & 0 & 676694 & 16828 & 0.4246 \\
\hline Taber & 1943 & 1.4 & 28138 & 12327 & 0.3946 \\
\hline
\end{tabular}


(8.421), acetic anhydride (8.309), petroleum ether (7.734), ethyl acetate (7.597), $\mathrm{N}, \mathrm{N}$-dimethyltoluidine (7.592), methyl tertbutyl ether (7.365), dichloromethane (7.129), methanol (5.426), and ethanol (4.773). The Lampman plan had the highest overall RSGI value of $112,106 \mathrm{~kg} / \mathrm{kg}$ vanillin due to the high masses of impactful solvents employed to obtain $1 \mathrm{~kg}$ of vanillin product, namely, nitrobenzene, diethyl ether, and cyclohexane.

\section{Ranking of vanillin plans}

Once numerical values of PMI, SR, IEE, RSGI, and SI are available for a given set of synthesis plans to a common target product, it is possible to use some kind of ranking algorithm to identify which ones have the highest overall performances based on these five attributes. From our previous work [20] we compared and contrasted the Borda positional counting method [21-24], established in 1781 by Jean-Charles de Borda (1733-1799), and the poset pairwise dominance analysis method [25] on 6 synthesis plans for the pharmaceutical apixaban. In implementing the Borda count method we first list the plans in ascending order of PMI, SR, IEE, and RSGI so that plans having the lowest values for these attributes are ranked highest; and we list the plans in descending order of SI so that plans having the highest values of SI are ranked highest. Since in this analysis there are 22 vanillin plans to consider the top ranking plan in any given list is assigned a point value of 22 and the lowest ranking plan is assigned a point value of 1 with all other plans having intermediate points accordingly. The maximum Borda count score corresponds to the number of plans considered in the set. In cases of plans having the same numerical values for a given attribute, they are assigned the same Borda count score. For example, for the SR attribute there are 10 plans that have an SR value of 0. Hence, each of them is assigned a Borda count score of 22 and the following lower ranking plans are successively given lower values in descending order. In this case the lowest ranking SR plan is given a Borda count score of 10 . Once all Borda count scores are determined for all plans for each attribute, the scores for each plan are added up and these summed scores are ranked from highest to lowest. Table 4 shows the net results of the Borda count method implemented on all 22 synthesis plans for vanillin considered. Part 3 of Supporting Information File 1 contains all of the Borda count data. We observe that the Givaudan-Roure (102) and Haarmann and Reimer (92) biofermentation routes have the highest overall ranking across the 5 attributes according to the Borda count method followed closely by the percolation extraction of whole vanilla beans (90), Faith synthesis from lignosul-

\begin{tabular}{|c|c|c|c|c|c|c|}
\hline $\begin{array}{l}\text { Alphabetized list of vanillin } \\
\text { synthesis plans }\end{array}$ & PMI points & SR points & IEE points & RSGI points & SI points & $\begin{array}{l}\text { overall Borda } \\
\text { count }\end{array}$ \\
\hline Borregaard synthesis & 18 & 19 & 11 & 22 & 14 & 84 \\
\hline Collins chemical & 19 & 21 & 12 & 20 & 11 & 83 \\
\hline Eilks Pt 1 & 8 & 11 & 17 & 10 & 13 & 59 \\
\hline Eilks Pt 2 & 16 & 22 & 19 & 17 & 3 & 77 \\
\hline Faith & 20 & 22 & 15 & 21 & 9 & 87 \\
\hline Frost & 3 & 12 & 9 & 8 & 19 & 51 \\
\hline Givaudan-Roure & 22 & 22 & 21 & 22 & 15 & 102 \\
\hline Haarmann and Reimer & 9 & 22 & 18 & 22 & 21 & 92 \\
\hline Hibbert & 12 & 13 & 4 & 11 & 7 & 47 \\
\hline Ji & 6 & 14 & 10 & 6 & 4 & 40 \\
\hline Lampman & 1 & 10 & 2 & 4 & 1 & 18 \\
\hline Lesage-Meesen & 2 & 16 & 5 & 22 & 22 & 67 \\
\hline Mayer & 21 & 18 & 16 & 19 & 2 & 76 \\
\hline Mexican group SFE & 4 & 22 & 3 & 5 & 16 & 50 \\
\hline Mottern & 17 & 22 & 20 & 18 & 10 & 87 \\
\hline Ontario Paper Co. & 13 & 15 & 7 & 14 & 12 & 61 \\
\hline percolation extraction cut & 11 & 22 & 22 & 12 & 18 & 85 \\
\hline percolation extraction whole & 10 & 22 & 22 & 16 & 20 & 90 \\
\hline Sorensen-Mehlum & 15 & 17 & 8 & 15 & 6 & 61 \\
\hline Soxhlet extraction cut & 14 & 22 & 13 & 13 & 17 & 79 \\
\hline Soxhlet extraction ground & 5 & 22 & 6 & 7 & 8 & 48 \\
\hline Taber & 7 & 20 & 14 & 9 & 5 & 55 \\
\hline
\end{tabular}

${ }^{a}$ Entries highlighted in bold represent the highest scores in each of the 5 attribute categories. 
fonic acid liquor (87), and Mottern four-step chemical synthesis from guaiacol (87). The lowest ranking plan was found to be the Lampman synthesis from sawdust, which had the highest overall process mass intensity (PMI $=32,540 \mathrm{~kg} / \mathrm{kg}$ ), second highest energy consumption requirements (IEE $=2,398,980 \mathrm{~kJ} / \mathrm{kg}$ ), fourth-highest solvent impact ranking $($ RSGI $=112,106 \mathrm{~kg} / \mathrm{kg})$, and lowest sustainability index value $(\mathrm{SI}=0.3676)$.

In implementing the poset pairwise dominance algorithm we determine the number of pairwise attributes and the number of pairwise plan comparisons for each pairwise attribute in order to determine the overall size of the ranking exercise. Since there are 5 attributes the number of pairwise attribute comparisons is $\mathrm{C}(5,2)=5 ! /((5-2) ! 2 !)=10$. The explicit list is as follows: PMI versus SR, PMI versus IEE, PMI versus RSGI, PMI versus SI, SR versus IEE, SR versus RSGI, SR versus SI, IEE versus RSGI, IEE versus SI, and RSGI versus SI. Since there are 22 synthesis plans for vanillin the number of pairwise plan comparisons is $C(22,2)=22 ! /((22-2) ! 2 !)=231$. Hence, there are overall $10 \times 231=2310$ pairwise comparisons that need to be made in the entire poset analysis. In general, a complete poset analysis on $K$ synthesis plans to a common target product based on $m$ attributes will require $\mathrm{C}(K, 2) * \mathrm{C}(m, 2)=(K ! /(K-2)$ ! $2 !) \cdot(m ! /(m-2) ! 2 !)$ pairwise comparisons. For a given pairwise plan comparison for a pair of attributes there are two possible outcomes: (a) a comparable pair in which plan A dominates plan B for both attributes $\mathrm{X}$ and $\mathrm{Y}$; and (b) an incomparable pair in which plan A dominates plan B for attribute $\mathrm{X}$ and plan $\mathrm{B}$ dominates plan A for attribute $Y$. For facile visual display of the results upper triangular $22 \times 22$ matrices are constructed showing green-coloured entries for comparable pairs and red- coloured entries for incomparable pairs. When a comparable pair for a given pairwise attribute comparison is found the dominant plan is identified. This sequence of steps is repeated for each pairwise attribute comparison and then the number of dominant occurrences for each plan are tallied up. Part 4 of Supporting Information File 1 summarizes the ten $22 \times 22$ matrices and the number of dominances for each plan for each pairwise attribute comparison. Supporting Information File 3 contains an Excel template file that facilitates carrying out the tedious task of pairwise comparisons involved in the poset analysis, particularly when the number of synthesis plans under consideration is large. Table 5 summarizes the main results of the poset dominance analysis for all vanillin plans considered. We observe that plans p7 (Givaudan-Roure), p8 (Haarmann and Reimer), and p18 (percolation extraction whole vanilla beans) have the highest number of pairwise dominances of 145 , 105, and 101, respectively. Plans p11 (Lampman synthesis from sawdust), p10 (Ji synthesis from guaiacol), p14 (Mexican group SFE), p9 (Hibbert synthesis from lignosulfonic acid liquor), p21 (Soxhlet extraction from ground vanilla beans), and p6 (Frost biofermentation from D-glucose) have the fewest number of dominances of $0,18,23,27,28$, and 29 , respectively. Table 6 and Table 7 summarize the results of the two ranking algorithms. Both methods identify the same set of overall best plans and overall worst plans with 11 out of the 22 plans having exactly the same ranking. With respect to plans having different ranking orders we find that 5 out of 22 plans have $+/-1$ rank positional change, 5 out of 22 plans have $+/-2$ rank positional change, and 1 plan out of 22 having a $+/-3$ rank positional change. Overall, the faster Borda count method is able to quickly identify the top and bottom performing plans with certainty. The more tedious poset pairwise dominance analysis

\begin{tabular}{|c|c|c|c|c|c|c|c|c|c|c|c|c|c|c|c|c|c|c|c|c|c|c|}
\hline \multirow{2}{*}{$\begin{array}{l}\text { pairwise } \\
\text { attribute } \\
\text { comparison }\end{array}$} & \multicolumn{22}{|c|}{ number of dominances } \\
\hline & $\mathrm{p} 1$ & p2 & p3 & p4 & p5 & p6 & p7 & p8 & p9 & p10 & $\mathrm{p} 11$ & p12 & p13 & p14 & p15 & p16 & p17 & p18 & p19 & p20 & p21 & p22 \\
\hline PMI vs SR & 8 & 10 & 1 & 9 & 10 & 1 & 12 & 6 & 3 & 2 & 0 & 1 & 8 & 3 & 9 & 5 & 6 & 6 & 7 & 8 & 3 & 4 \\
\hline PMI vs IEE & 9 & 10 & 7 & 13 & 11 & 8 & 19 & 2 & 3 & 5 & 0 & 1 & 14 & 1 & 9 & 4 & 9 & 9 & 6 & 8 & 3 & 6 \\
\hline PMI vs RSGI & 17 & 16 & 5 & 12 & 15 & 1 & 21 & 8 & 7 & 2 & 0 & 1 & 15 & 1 & 14 & 9 & 7 & 7 & 11 & 9 & 2 & 5 \\
\hline PMI vs SI & 10 & 9 & 4 & 1 & 7 & 1 & 14 & 7 & 3 & 1 & 0 & 1 & 1 & 1 & 7 & 5 & 6 & 6 & 3 & 7 & 1 & 2 \\
\hline SR vs IEE & 7 & 8 & 1 & 12 & 9 & 1 & 12 & 12 & 1 & 3 & 0 & 2 & 7 & 3 & 12 & 2 & 13 & 13 & 4 & 9 & 4 & 8 \\
\hline SR vs RSGI & 8 & 9 & 1 & 9 & 9 & 1 & 11 & 11 & 3 & 1 & 0 & 6 & 7 & 1 & 8 & 5 & 6 & 8 & 6 & 6 & 2 & 3 \\
\hline SR vs SI & 7 & 5 & 1 & 2 & 7 & 2 & 10 & 11 & 1 & 1 & 0 & 5 & 1 & 10 & 6 & 4 & 9 & 11 & 2 & 10 & 6 & 3 \\
\hline IEE vs RSGI & 9 & 8 & 6 & 11 & 10 & 3 & 18 & 15 & 2 & 1 & 0 & 3 & 10 & 1 & 11 & 3 & 8 & 11 & 5 & 6 & 3 & 5 \\
\hline IEE vs SI & 7 & 5 & 10 & 2 & 5 & 7 & 14 & 15 & 1 & 1 & 0 & 3 & 1 & 1 & 9 & 3 & 17 & 18 & 1 & 9 & 2 & 2 \\
\hline RSGI vs SI & 13 & 8 & 3 & 1 & 8 & 4 & 14 & 18 & 3 & 1 & 0 & 18 & 1 & 1 & 8 & 5 & 7 & 12 & 3 & 7 & 2 & 2 \\
\hline TOTALS & 95 & 88 & 39 & 72 & 91 & 29 & 145 & 105 & 27 & 18 & 0 & 41 & 65 & 23 & 93 & 45 & 88 & 101 & 48 & 79 & 28 & 40 \\
\hline
\end{tabular}

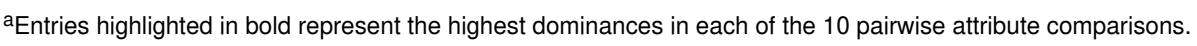


Table 6: Summary of Borda count and poset dominance analysis of 22 synthesis plans for vanillin listed alphabetically.

\begin{tabular}{llll} 
Alphabetized list of vanillin synthesis plans & plan code & Borda count & poset dominances \\
\hline Borregaard synthesis & p1 & 84 & 95 \\
Collins chemical & p2 & 83 & 88 \\
Eilks Pt 1 & p3 & 59 & 39 \\
Eilks Pt 2 & p4 & 77 & 72 \\
Faith & p5 & 87 & 91 \\
Frost & p6 & 51 & 29 \\
Givaudan-Roure & p7 & 102 & 145 \\
Haarmann and Reimer & p8 & 92 & 105 \\
Hibbert & p9 & 47 & 27 \\
Ji & p10 & 40 & 18 \\
Lampman & p11 & 18 & 0 \\
Lesage-Meesen & p12 & 41 \\
Mayer & p13 & 67 & 65 \\
Mexican group SFE & p14 & 76 & 23 \\
Mottern & p15 & 50 & 93 \\
Ontario Paper Co. & p16 & 87 & 45 \\
percolation extraction cut & p17 & 61 & 88 \\
percolation extraction whole & p18 & 85 & 101 \\
Sorensen-Mehlum & p19 & 90 & 48 \\
Soxhlet extraction cut & p20 & 61 & 79 \\
Soxhlet extraction ground & p21 & 79 & 28 \\
Taber & p22 & 48 & 40 \\
\end{tabular}

Table 7: Summary of Borda count and poset dominance rankings of 22 synthesis plans for vanillin. ${ }^{\text {a,b }}$

\begin{tabular}{|c|c|c|c|}
\hline Borda count ranking & plan & Poset dominance ranking & plan \\
\hline 102 & p7 & 145 & p7 \\
\hline 92 & p8 & 105 & $p 8$ \\
\hline 90 & p18 & 101 & p18 \\
\hline 87 & p5 & 95 & p1 \\
\hline 87 & $p 15$ & 93 & p15 \\
\hline 85 & p17 & 91 & p5 \\
\hline 84 & p1 & 88 & p2 \\
\hline 83 & p2 & 88 & p17 \\
\hline 79 & $p 20$ & 79 & $p 20$ \\
\hline 77 & p4 & 72 & p4 \\
\hline 76 & $p 13$ & 65 & p13 \\
\hline 67 & p12 & 48 & p19 \\
\hline 61 & $p 16$ & 45 & p16 \\
\hline 61 & p19 & 41 & p12 \\
\hline 59 & p3 & 40 & p22 \\
\hline 55 & p22 & 39 & p3 \\
\hline 51 & p6 & 29 & p6 \\
\hline
\end{tabular}


Table 7: Summary of Borda count and poset dominance rankings of 22 synthesis plans for vanillin. ${ }^{\text {a,b }}$ (continued)

\begin{tabular}{llll}
50 & $\mathbf{p 1 4}$ & 28 & $\mathbf{p 2 1}$ \\
48 & $\mathbf{p 2 1}$ & 27 & $\mathbf{p 9}$ \\
47 & $\mathbf{p 9}$ & 23 & $\mathbf{p 1 4}$ \\
\hline 40 & $p 10$ & 18 & $p 10$ \\
18 & $p 11$ & 0 & $p 11$
\end{tabular}

aLine separated, italicized entries represent plans having exactly the same ranking order. ${ }^{b}$ Bold entries represent plans having different ranking orders.

is more reliable in ranking the intermediate performing plans due to its thoroughness in considering all possible pairwise plan comparisons across all attributes considered.

\section{Conclusion}

We have introduced and demonstrated how a sustainability index (SI) can be computed specifically for synthesis plans based on the provenance of input materials and energy sources, and the fate of output waste materials. Reasonable and reliable estimates of SI based on provenance and fate of input and output materials respectively can only be made if full disclosure of both supply and disposal chains in the chemical enterprise exists. We note that this is a formidable challenge for the chemical community to accept and adopt in routine practice. We also note that the computation of SI will necessarily involve significant assumptions to be made in determining key parameters such as $F_{R E}$ and $F_{V P}$ and that these assumptions, in turn, will necessarily affect the ranking of synthesis plans. The nine assumptions listed for the analysis of vanillin are an illustrative example of what is entailed for the computation of SI. Other target products will require their own set of assumptions. Nevertheless, we believe that the protocols disclosed in this work are easily implementable once the necessary data are made available.

In our determination of the four fractions contributing to SI we implemented a binary approach based on whether or not a material or energy input arises from renewable or non-renewable sources; and on whether or not an output material could be recycled or reused. Specific rates of depletion versus renewal of a resource applied to inputs and specific rates of reusability versus accumulation of outputs require a complete macroscopic knowledge and connectivity of all elements pertaining to the network of all chemical processes involved in a given synthesis plan. At this time reliable estimates of these rates are not readily available to the average practicing chemist or chemical engineer in established open-access data collection databases for all commodity material resources, and so this significant limitation prevents estimation of time analyses pertaining to how long a given resource may exist under a so-called "sustainability condition". For first generation chemical feedstocks arising from fossil fuels, the rate of finding new reserves of fossil fuel may be used as a rate of "renewal" rather than the geological rate of renewal which is several orders of magnitude lower. In any case, rates of finding new reserves of fossil fuels or mineral deposits depend on knowledge of counting existing reserves, which necessarily requires reliable databases that constantly track data annually. Such tracking is not always in industry's best interest to disclose such information publicly for economic and political reasons. For example, providing inaccurate or incomplete information to governments and investors can leverage control of prices of crude oil, natural gas, and metals; whereas, disclosing accurate and up-to-date information can expose vulnerabilities among governments and investors that can be taken advantage of.

Furthermore, deciding where to terminate a chain of resources, i.e., which material to designate as the "starting material" for a given synthesis plan, remains a non-resolvable dilemma. What is known with certainty is that extending a starting material chain will necessarily amplify material consumption (PMI), enthalpic energy inputs (IEE), and associated environmental and safety-hazard impacts (RSGI). Hence, if the starting points of the vanillin plans in this work are changed, particularly for the chemical syntheses, then this will change the values of all of these parameters and ultimately the ranking order of the plans based on those parameters. There is also the problem of including the syntheses of all reagents and catalysts used in each reaction step involved in a given synthesis plan. Again, significant amplification of material consumption (PMI), enthalpic energy inputs (IEE), and associated environmental and safety-hazard impacts (RSGI) will result. For the case of $F_{V I}$ which is the ratio of mass of valorized input to mass of total input, if we were to include the synthesis route of a catalyst used in the main synthesis chain we can envisage two case scenarios for how this will impact the computation of $F_{V I}$ where the mass of total inputs (denominator) will obviously increase. In one case, if the mass of inputs to make the catalyst is considered valorized, then the numerator magnitude will also increase. Hence, the overall value of $F_{V I}$ is expected to increase which will in turn increase 
the value of SI. On the other hand, if the mass of inputs to make the catalyst is considered non-valorized, then the numerator magnitude does not change. Hence, the overall value of $F_{V I}$ is expected to decrease which will in turn decrease the value of SI. Similar trends apply for $F_{V O}$ and $F_{R E}$. Such a scenario will have a negative impact on chemical syntheses that primarily use nonrenewable materials, reagents, and catalysts. The net effect is to drive down the ranking of chemical syntheses. Chemical syntheses have a lower likelihood of achieving moderate levels of sustainability compared to ones based on biofeedstocks and biofermentation processes mainly because they utilize fossil fuel-derived materials. In any event, the task of tracing starting materials, catalysts, and reaction solvent syntheses is very tedious, especially for time-pressed chemists who wish to practice green chemistry. However, such a task can be significantly alleviated if synthesis databases of first, second, and third generation feedstocks existed where all key metrics have been worked out in advance such as PMI, SR, IEE, and RSGI. It is then possible to tap into these databases in a cassette-like manner and insert these synthesis chain extensions to the main synthesis chain of interest as needed. The creation of such databases requires significant investment in time and energy but once done it is expected that they will have far-reaching utility in the long run. An important point to keep in mind in extending synthesis chains to first generation feedstocks of simple chemical complexity is that environmental and safety-hazard impacts of reactants and reagents become important since reaction solvent usage dramatically decreases. Reactions involving first generation feedstocks are typically gas-phase reactions run without any reaction solvent. However, as the synthesis chain extends to more complex intermediates and other materials, reaction solvent usage increases dramatically and becomes the bulk input mass of materials used. Hence, environmental and safety-hazard impacts of reaction solvents become important in the pharmaceutical industry, for example.

Deciding on where to terminate a chain is a contentious issue; however, general guidelines can be created to help direct and facilitate decision-making in the form of a decision tree. A first key question to ask is: "Does a plan trace to a renewable or reclaimed starting material?". If the answer is "yes", then all metrics analyses up to that renewable material need to be done. On the other hand, if the answer is "no", then there are three possible options in decreasing order of thoroughness. The first option is to trace to a non-renewable starting material that is common to all synthesis plans compared as far as possible. The second option, if no common starting material can be found, is to trace to a non-renewable starting material (i) that is a first generation feedstock such as coal, crude oil, or ores from the earth's crust; or (ii) whose molecular weight is less than $80 \mathrm{~g} / \mathrm{mol}$ corresponding to benzene or pyridine starting materi- als. The third option is to trace to a "readily available" or commercially available starting material. An agreed consensus between academic, industry, and government stakeholders is needed to decide which option is most appropriate and feasible, or to decide other options. The main criterion for tracing each reaction step in a backwards fashion is to always choose literature examples with the least PMI, highest yields and atom economies, and least environmental-safety hazard impacts. As one goes towards virgin materials energy demands and hazard impacts generally increase. All synthesis plans should be compared in the same way to avoid biased ranking. For example, if reagents and catalysts are not traced further back, then this is done for all plans considered to a given target product. On the other hand, if they are traced back to earlier materials then this should be done for all plans. A key problem is that ranking positions can be arbitrarily selected for a given set of synthesis plans to a common target product simply by selecting the cutoff chain of starting materials. Hence, the computation of SI and subsequent ranking are most vulnerable to the apparent arbitrary choice of starting material cut-offs in the analyses. Transportation costs for getting starting materials to the manufacturing site also contribute to input energy; however, for the case of simplicity we have not considered these in the present analysis.

Based on the above challenges and limitations discussed above, we believe that our choice of starting materials used to carry out our metric analyses for all synthesis plans of vanillin considered as shown in Figure S1 (Supporting Information File 1) are reasonable and lead to fair ranking comparisons. We have also shown how synthesis plans may be compared and ranked using Borda count and poset pairwise dominance algorithms according to the following attributes: process mass intensity (PMI), sacrificial reagent consumption (SR), input enthalpy energy consumption (IEE), Rowan solvent greenness index (RSGI), and sustainability index (SI). The Borda count method is found to be adequate for rapid identification of best and worst plans in a given set of synthesis plans, whereas, the more detailed poset pairwise dominance analysis is appropriate for obtaining a precise ranking of intermediate performing plans. Application of these ranking methods to 22 synthesis plans for vanillin indicated that biofermentation processes from ferulic acid and isoeugenol starting materials were best performers overall followed closely by percolation solvent extraction processes from whole or cut cured vanilla beans. Chemical syntheses of vanillin from lignosulfonic acid liquors or guaiacol were surprisingly competitive when analyzed according to all of the 5 comparative attributes; however, they ranked low with respect to the sustainability index alone. The most sustainable processes were biofermentations and percolation solvent extractions. 
Table 8: Abbreviations

\begin{tabular}{|c|c|}
\hline Abbreviation & Explanation \\
\hline ABP & acidification-basification potential \\
\hline aqua & aquatic toxicity, $\mathrm{mg} / \mathrm{L}$ for $96 \mathrm{~h}$ \\
\hline $\mathrm{BCP}$ & bioconcentration potential, unitless \\
\hline$F_{V I}$ & mass fraction of valorized inputs \\
\hline$F_{N V I}$ & mass fraction of non-valorized inputs \\
\hline$F_{V O}$ & mass fraction of valorized outputs \\
\hline$F_{N V O}$ & mass fraction of non-valorized outputs \\
\hline$F_{R E}$ & $\begin{array}{l}\text { input enthalpic energy fraction arising from } \\
\text { renewable energy sources }\end{array}$ \\
\hline$F_{V P}$ & mass fraction of valorized target product \\
\hline FP & flash point, degrees $\mathrm{K}$ \\
\hline GWP & global warming potential, unitless \\
\hline IEE & input enthalpic energy, kJ per kg product \\
\hline K & $\begin{array}{l}\text { number of synthesis plans to a common } \\
\text { target product }\end{array}$ \\
\hline $\mathrm{LC}_{50}$ & $\begin{array}{l}\text { lethal concentration required to kill } 50 \% \text { of } \\
\text { population, } \mathrm{g} / \mathrm{m}^{3} \text { for } 4 \mathrm{~h}\end{array}$ \\
\hline $\mathrm{LD}_{50}$ & $\begin{array}{l}\text { lethal dose required to kill } 50 \% \text { of population, } \\
\mathrm{mg} / \mathrm{kg} \text { body weight }\end{array}$ \\
\hline$m$ & $\begin{array}{l}\text { number of attributes used in a ranking } \\
\text { algorithm }\end{array}$ \\
\hline$M$ & metric \\
\hline$M_{V I}$ & $\begin{array}{l}\text { mass of valorized or renewably sourced } \\
\text { inputs }\end{array}$ \\
\hline$M_{N V I}$ & $\begin{array}{l}\text { mass of non-valorized or non-renewably } \\
\text { sourced inputs }\end{array}$ \\
\hline$M_{\text {product }}$ & $\begin{array}{l}\text { mass of target product produced in a } \\
\text { synthesis plan }\end{array}$ \\
\hline$M_{\text {product }}^{*}$ & $\begin{array}{l}\text { mass of target product that is destined to be } \\
\text { waste after its intended use }\end{array}$ \\
\hline ODP & ozone depletion potential, unitless \\
\hline OEL & occupational exposure limit, ppm \\
\hline OSI & overall solvent index \\
\hline PER & persistence potential, unitless \\
\hline PMI & $\begin{array}{l}\text { process mass intensity, } \mathrm{kg} \text { total inputs per } \mathrm{kg} \\
\text { product }\end{array}$ \\
\hline Q & quotient referring to risk phrases, unitless \\
\hline$r$ & rank number for a plan \\
\hline RE & renewable energy \\
\hline RSGI & $\begin{array}{l}\text { Rowan solvent greenness index, } \mathrm{kg} \text { per } \mathrm{kg} \\
\text { product }\end{array}$ \\
\hline SD & skin dose, mg \\
\hline SFE & supercritical fluid extraction \\
\hline SFP & smog forming potential, unitless \\
\hline SI & sustainability index, dimensionless \\
\hline SR & sacrificial reagents \\
\hline$w_{V O}$ & $\begin{array}{l}\text { mass of waste of valorized or reusable } \\
\text { outputs }\end{array}$ \\
\hline$W_{N V O}$ & mass of waste of non-valorized outputs \\
\hline$W_{\text {total }}$ & $\begin{array}{l}\text { total mass of waste produced in a synthesis } \\
\text { plan }\end{array}$ \\
\hline
\end{tabular}

\section{Supporting Information}

\section{Supporting Information File 1}

Figure $\mathrm{S} 1$ comprising 22 vanillin synthesis plans, data for vanillin synthesis plans on production of $1 \mathrm{~kg}$ vanillin,

Borda count results, and Poset analysis.

[https://www.beilstein-journals.org/bjoc/content/ supplementary/1860-5397-16-196-S1.pdf]

\section{Supporting Information File 2}

Excel file of sustainability index (SI) calculator. [https://www.beilstein-journals.org/bjoc/content/ supplementary/1860-5397-16-196-S2.xls]

\section{Supporting Information File 3}

Excel template file for carrying out poset pairwise dominance analysis.

[https://www.beilstein-journals.org/bjoc/content/ supplementary/1860-5397-16-196-S3.xls]

\section{Acknowledgements}

This paper is dedicated to the memory of Christopher R. Schmid, founding editor of Organic Process Research and Development and process chemist at Eli Lilly, who understood the true meaning and scope of both green chemistry and sustainability chemistry, and who foresaw their practical applications widely in the chemical industry. Facundo Jesus Salamanca from Don Mills Collegiate Institute Mathematics Club is thanked for checking the mathematical treatment.

\section{ORCID ${ }^{\circledR}$ iDs}

John Andraos - https://orcid.org/0000-0002-2982-2708

\section{References}

1. Hogue, C. Chem. Eng. News 2019, 97, 19.

2. Hinderink, A. P.; van der Kooi, H. J.; de Swaan Arons, J. Green Chem. 1999, 1, G176-G180. doi:10.1039/a909915h

3. Dewulf, J.; Van Langenhove, H.; Mulder, J.; van den Berg, M. M. D.; van der Kooi, H. J.; de Swaan Arons, J. Green Chem. 2000, 2, 108-114. doi:10.1039/b000015i

4. Lems, S.; van der Kooi, H. J.; de Swaan Arons, J. Green Chem. 2002, 4, 308-313. doi:10.1039/b203490p

5. Cséfalvay, E.; Akien, G. R.; Qi, L.; Horváth, I. T. Catal. Today 2015, 239, 50-55. doi:10.1016/j.cattod.2014.02.006

6. Horváth, I. T.; Cséfalvay, E.; Mika, L. T.; Debreczeni, M. ACS Sustainable Chem. Eng. 2017, 5, 2734-2740. doi:10.1021/acssuschemeng.6b03074

7. Cséfalvay, E.; Horváth, I. T. ACS Sustainable Chem. Eng. 2018, 6, 8868-8874. doi:10.1021/acssuschemeng.8b01213

8. Sikdar, S. K. AIChE J. 2003, 49, 1928-1932. doi:10.1002/aic.690490802 
9. Sheldon, R. A.; Sanders, J. P. M. Catal. Today 2015, 239, 3-6. doi:10.1016/j.cattod.2014.03.032

10. Fadel, C.; Tarabieh, K. Resources 2019, 8, 115. doi:10.3390/resources8020115

11. Provenance. https://en.wikipedia.org/w/index.php?title=Provenance \&oldid=9183602 47 (accessed Sept 30, 2019).

12. McElroy, C. R.; Constantinou, A.; Jones, L. C.; Summerton, L.; Clark, J. H. Green Chem. 2015, 17, 3111-3121. doi:10.1039/c5gc00340g

13. Jimenez-Gonzalez, C.; Ponder, C. S.; Broxterman, Q. B.; Manley, J. B. Org. Process Res. Dev. 2011, 15, 912-917. doi:10.1021/op200097d

14. Redlich, O.; Kwong, J. N. S. Chem. Rev. 1949, 44, 233-244. doi:10.1021/cr60137a013

15. Ruiz-Mercado, G. J.; Smith, R. L.; Gonzalez, M. A. Ind. Eng. Chem. Res. 2012, 51, 2309-2328. doi:10.1021/ie102116e

16. Ontario's System-Wide Electricity Supply Mix: 2017 Data. https://www.oeb.ca/sites/default/files/2017-supply-mix-data.pdf (accessed Sept 30, 2019).

17. Archived - 2017 Long-Term Energy Plan: Discussion Guide. https://www.ontario.ca/document/2017-long-term-energy-plan-discussio n-guide/ontarios-energy-mix-end-2015 (accessed Sept 30, 2019).

18. Trost, B. M. Science 1991, 254, 1471-1477. doi:10.1126/science. 1962206

19. Slater, C. S.; Savelski, M. J. Environ. Sci. Health, Part A: Toxic Hazard. Subst. Environ. Eng. 2007, 42, 1595-1605. doi:10.1080/10934520701517747

20. Andraos, J. Green Process. Synth. 2019, 8, 787-801. doi:10.1515/gps-2019-0048

21. de Borda, J. C. Mémoire de l'Académie Royale. Histoire de l'Académie des Sciences, 1781, 657-665. http://gerardgreco.free.fr/IMG/pdf/MA_c_moire-Borda-1781.pdf (accessed Sept 30, 2019).

22. Emerson, P. Soc. Choice Welfare 2013, 40, 353-358. doi:10.1007/s00355-011-0603-9

23. Levin, J.; Nalebuff, B. J. Econ. Perspect. 1995, 9, 3-26. doi:10.1257/jep.9.1.3

24. Saari, D. G. The Optimal Ranking Method Is the Borda Count; International Institute for Applied Systems Analysis: Laxenburg, Austria, 1985

25. Restrepo, G.; Stadler, P. F. ACS Sustainable Chem. Eng. 2016, 4 , 2191-2199. doi:10.1021/acssuschemeng.5b01649

26. Li, K.; Frost, J. W. J. Am. Chem. Soc. 1998, 120, 10545-10546. doi:10.1021/ja9817747

27. Muheim, A.; Müller, B.; Münch, T.; Wetli, M. Process for the production of vanillin. Eur. Pat. Appl. EP885968 A1, Dec 23, 1998.

28. Lesage-Meessen, L.; Delattre, M.; Haon, M.; Thibault, J.-F.; Ceccaldi, B. C.; Brunerie, P.; Asther, M. J. Biotechnol. 1996, 50, 107-113. doi:10.1016/0168-1656(96)01552-0

29. Lesage-Meesen, L.; Delattrea, M.; Haona, M.; Asther, M. Aspergillus niger which produces vanillic acid from ferulic acid. U.S. Patent US6162637, Dec 19, 2000.

30. Rabenhorst, J.; Hopp, R. Process for the preparation of vanillin. U.S. Patent US5017388, May 21, 1991.

31. Faith, W. L.; Keyes, D. B.; Clark, R. L. Industrial Chemicals, 3rd ed.; Wiley: New York, NY, USA, 1966; $p 796$.

32. Bjørsvik, H.-R.; Minisci, F. Org. Process Res. Dev. 1999, 3, 330-340. doi:10.1021/op9900028

33. Tomlinson, G. H., 2nd.; Hibbert, H. J. Am. Chem. Soc. 1936, 58, 345-348. doi:10.1021/ja01293a046
34. Hibbert, H.; Tomlinson, G. H. Manufacture of vanillin from waste sulphite pulp liquor. U.S. Patent US2069185, Jan 26, 1937.

35. Craig, D.; Logan, C. D. Method of producing vanillin and other useful products from lignosulfonic acid compounds. U.S. Patent US3054659, Sept 18, 1962.

36. Lampman, G. M.; Andrews, J.; Bratz, W.; Hanssen, O.; Kelley, K.; Perry, D.; Ridgeway, A. J. Chem. Educ. 1977, 54, 776-778. doi:10.1021/ed054p776

37. Sorensen, N. A.; Mehlum, J. Method of manufacturing vanillin. U.S. Patent US2752394, June 26, 1956.

38. Mayer, E. Oesterr. Chem.-Ztg. 1949, 50, 40-41.

39. Mottern, H. O. J. Am. Chem. Soc. 1934, 56, 2107-2108. doi:10.1021/ja01325a033

40. Fiecchi, A.; Mario, G.; Cabella, P.; Cicognani, G. Method of preparing vanillin from eugenol. U.S. Patent US3544621, Dec 1, 1970.

41. Garner, N.; Siole, A.; Eilks, I. J. Sci. Educ. 2016, 17, 25-28.

42. Huang, W.-B.; Du, C.-Y.; Jiang, J.-A.; Ji, Y.-F. Res. Chem. Intermed. 2013, 39, 2849-2856. doi:10.1007/s11164-012-0804-6

43. Taber, D. F.; Patel, S.; Hambleton, T. M.; Winkel, E. E. J. Chem. Educ. 2007, 84, 1158. doi:10.1021/ed084p1158

44. Sujalmi, S.; Suharso, S.; Supriyanto, R.; Buchari, B. Indones. J. Chem. 2010, 5, 7-10. doi:10.22146/ijc.21831

45. Sinha, A. K.; Sharma, U. K.; Sharma, N. Int. J. Food Sci. Nutr. 2008, 59, 299-326. doi:10.1080/09687630701539350

46. Castillo-Ruz, M. C.; Guillermo-Alcocer, C. G.; Bojorquez-Gamboa, R. R.; Rocha-Uribe, J. A. Tecnol., Cienc., Educ. 2011, 26, 80-84.

47. Couteau, D.; Mathaly, P. Ind. Crops Prod. 1997, 6, 237-252. doi:10.1016/s0926-6690(97)00014-9

48. Kaufman, T. S. J. Braz. Chem. Soc. 2015, 26, 1055-1086. doi:10.5935/0103-5053.20150086

49. Parfitt, J.; Barthel, M.; Macnaughton, S. Philos. Trans. R. Soc., B 2010, 365, 3065-3081. doi:10.1098/rstb.2010.0126

50. One Third of All Food Wasted! United Nations Regional Information Centre for Western Europe.

https://www.unric.org/en/food-waste/27133-one-third-of-all-food-wasted (accessed Sept 30, 2019).

51. Gustavsson, J.; Cederberg, C.; Sonesson, U.; van Otterdijk, R.; Meybeck, A. Global Food Losses and Food Waste-Extent, Causes, and Prevention. Food and Agriculture Organization of the United Nations, Rome, 2011. http://www.fao.org/3/a-i2697e.pdf (accessed Sept 30, 2019).

52. Lipinski, B.; Hanson, C.; Lomax, J.; Kitinoja, L.; Waite, R.; Searchinger, T. Reducing Food Loss and Waste. Working Paper, Installment 2 of Creating a Sustainable Food Future. Washington, DC: World Resources Institute, 2013.. https://pdf.wri.org/reducing_food_loss_and_waste.pdf (accessed Sept 30, 2019).

53. S Department of Agriculture. Food Waste FAQs. https://www.usda.gov/foodwaste/faqs (accessed Sept 30, 2019). 


\section{License and Terms}

This is an Open Access article under the terms of the Creative Commons Attribution License (https://creativecommons.org/licenses/by/4.0). Please note that the reuse, redistribution and reproduction in particular requires that the authors and source are credited.

The license is subject to the Beilstein Journal of Organic Chemistry terms and conditions:

(https://www.beilstein-journals.org/bjoc)

The definitive version of this article is the electronic one which can be found at:

https://doi.org/10.3762/bjoc.16.196 